\title{
Ultralight Last Mile Vehicles on Public Walkways for Sustainable Traffic Chains
}

\author{
Oliver Zirn ${ }^{1}$, Konrad Sagert ${ }^{2}$, Markus Rüther ${ }^{3}$ \\ ${ }^{1}$ University of Applied Sciences Esslingen, Faculty Automotive Systems \\ Kanalstraße 33, 73728 Esslingen, Germany \\ ${ }^{2}$ Fraunhofer IAO - Anwendungszentrum KEIM \\ Flandernstraße 101, 73732 Esslingen, Germany \\ ${ }^{3}$ Hudora GmbH, Jägerwald 15, 42897 Remscheid, Germany \\ oliver.zirn@hs-esslingen.de
}

\begin{abstract}
Foldable ultralight electric vehicles (E-ULV) with a range of 4-5 kilometers and a weight of 5-7 kilograms are a promising key component for future sustainable traffic chains in urban regions with mountainous topography. While E-Bikes are hard to use in the rush-hour of metropolitan public transport, these - actually not available and not legally usable - E-ULV are a real innovation for the "last mile". This paper presents electric drive add-on kits for foldable scooters that can be used on public walkways, train stations and subway platforms. The key innovation of these very light, slow and powerful foldable scooters is the adaptive speed limitation based on ultrasonic sensors, that limits the maximum velocity to $6 \mathrm{~km} / \mathrm{h}$ on crowded walkways and permits a faster movement up to $10 \mathrm{~km} / \mathrm{h}$ if the walkway is obstacle-free. The E-ULV had been tested legally in small fleets on public walkways in Stuttgart and Esslingen by special permission of the Ministry for Traffic and Infrastructure Baden-Württemberg. The modal split of the probands has been monitored by a specially designed Smartphone-App. These E-ULV turned out shift car kilometers to the public transport system effectively as well as they avoid parking lot search traffic.
\end{abstract}

Keywords: urban walkway traffic, personal light vehicle, electrical assistance

\section{Introduction}

Foldable scooters and bicycles are well known for decades. In flat metropolitan regions like Berlin, Hamburg or Cologne a light foldable vehicle is often used instead of walking, using a bicycle between the apartment or working place, the tramway or train station. In mountainous metropolitan regions like Zürich or Stuttgart, vehicles without additional power assistance are exhausting on longer climbs. Long downhill distances are a real challenge for foldable scooters. The typical rear wheel friction brake is effective for emergency stops as well as for short slopes. On longer slopes the friction power leads to a melting shoe sole. Although several electrical power assisted folding bikes (so called foldingPEDELEC's) are available on the marked, these bikes are quite heavy ( $>15 \mathrm{~kg})$, extremely expensive or of questionable quality. Moreover, all premium folding bike manufacturers have withdrawn their electrified versions in the last few years. Electrified folding scooters are available from Asian manufacturers (cheap and heavy) and latterly from Switzerland (lightweight and expensive) - all together much to fast (up to $25 \mathrm{~km} / \mathrm{h}$ ) and with no legal admission for middle European public roads or walkways. The Esslingen and Stuttgart Universities of Applied Sciences research cooperation "TrottiElec" developed an electric drive add-on kit for lightweight slow folding scooters with high gradability on public walkways (see chapter 2). Furthermore, the research cooperation obtained the support of the Ministry for Traffic and Infrastructure BadenWürttemberg (MVI-BW) for a temporary legal test fleet and developed a Smartphone-App (see chapter 3) to find out the shift of the modal split due to these new key elements. The main objective of this fleet experiment is the scientific sustainability and acceptance proof for E-ULV to initiate a suitable vehicle category and suitable product developments in middle Europe (see chapter 4). 


\section{Electrical Power Assisted Folding Scooters}

Folding scooters without power assistance are considered as toys and are tolerated by the German traffic authorities on public walkways. Their usage on public streets however is prohibited. Electrified scooters are power-driven vehicles and thus not allowed on walkways with the exception of handicapped aids (third wheel, seat, handicapped ID). Electrified scooters for public street usage require a type approval as well as a vehicle category. The type approval is actually only achievable for bicycle-like scooters (heavy and large). A vehicle category does not exist at the moment although there are efforts on European Union level to introduce a "Personal Light Electric Vehicle (PLEV)"-category for the street [1].
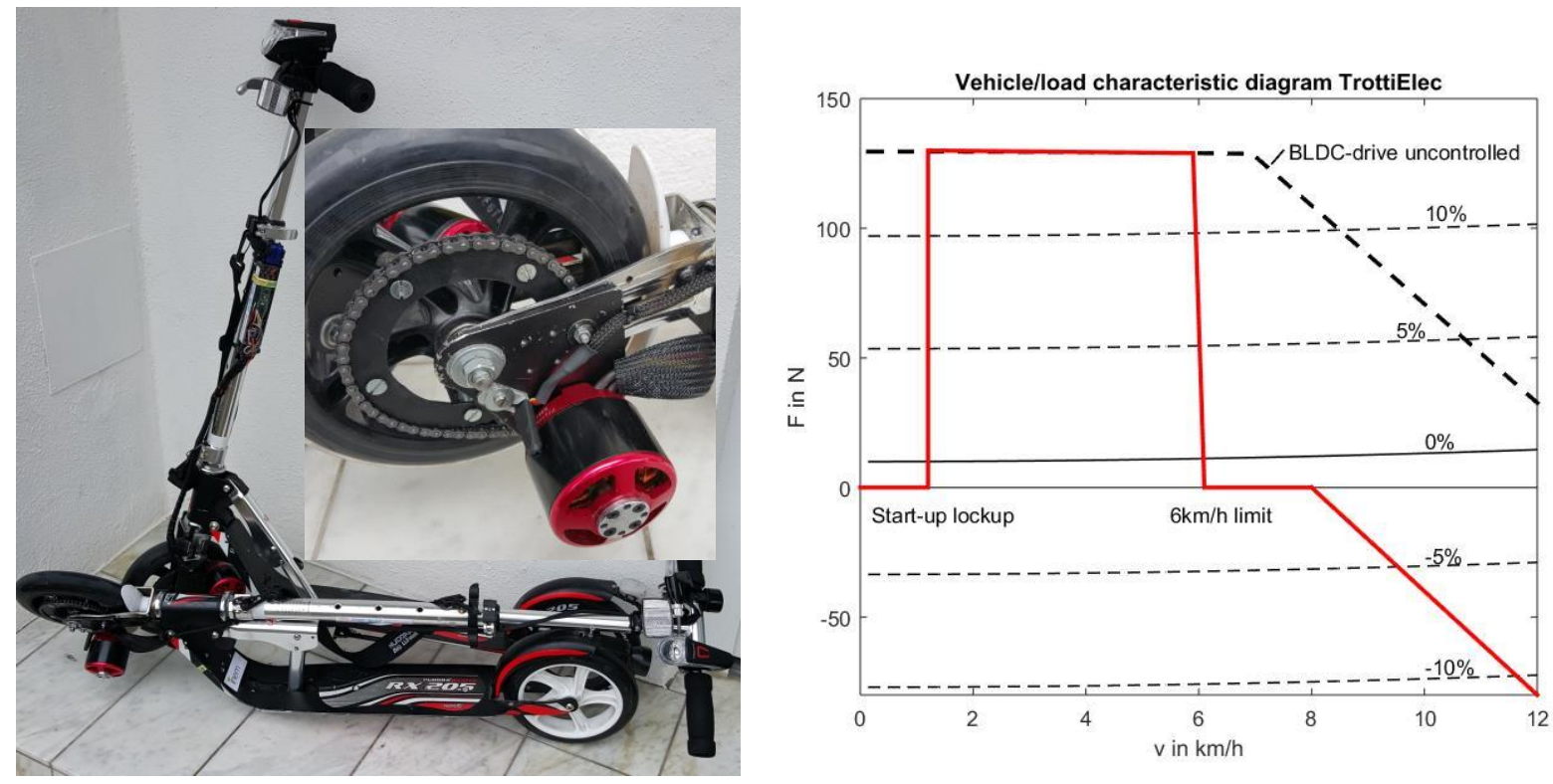

Fig. 1: Electrified folding scooters, front wheel drive chain (ca. $250 €, 1,3 \mathrm{~kg}$ additional weight) and vehicle characteristic diagram.

However, an electrified scooter for closed public transportation chains should be usable on walkways or train stations. A thoroughgoing discussion with the sustainable mobility department of the MVI-BW turned out the following preconditions for a time-limited admission of a test fleet in Stuttgart and Esslingen:

- $6 \mathrm{~km} / \mathrm{h}$ maximum velocity for electric power assistance, Tamper-proof drive chain

- Kicking assistance (comparable to the pedaling assistance of EPAC's)

- Dead-man-switch (drive stops, when the vehicle is lost or the driver is hamstrung)

- Friction brake and recuperation brake

- Vehicle identification number (FIN), no admission or type approval, no driving license or minimum age required

- Deviant from $\$ 2 \mathrm{StVO}$ public walkways have to be used, local traffic authority has to permit the fleet test area

Fig. 1 shows the drive of the add-on kit that fulfills the above mentioned preconditions [2,3]. The BLDC-Outrunner motor and the BLDC-power electronics are controlled by a microcontroller. The user input (thumb switch and brake lever) is transposed into the vehicle characteristic diagram shown in Fig. 1. Velocity and acceleration are measured by a hall switch in the stray magnetic field of the outrunner. The software kick sensor enables the electric assistance if a minimum acceleration is detected and holds this enable status for several seconds. The start-up lockup requires a certain minimum velocity to prevent misuse or mechanical injuries at the driven front wheel. All drive and brake set points are low-pass filtered for a smooth assistance/brake performance.

The Technical Supervisory Association (TÜV-Süd, Munich/Pforzheim) inspected the first prototypes and confirmed their conformity to the MVI-BW obligations (certificate no. 6230462318). Based on this certificate, the local traffic authorities in Esslingen and Stuttgart support a 2-year fleet experiment on public walkways. The first fleet with 25 TrottiElecs, non-motorised folding scooters and a pedestrian comparison group started in summer 2016. 


\section{Recording of Traffic Chains}

To collect the modal split and the traffic chain data (position, velocity, selected vehicles, weather conditions) a Smartphone-App has been developed by the Fraunhofer-IAO-KEIM applied research group, the so called "Mobility Recognizer", that is shown in figure 2.
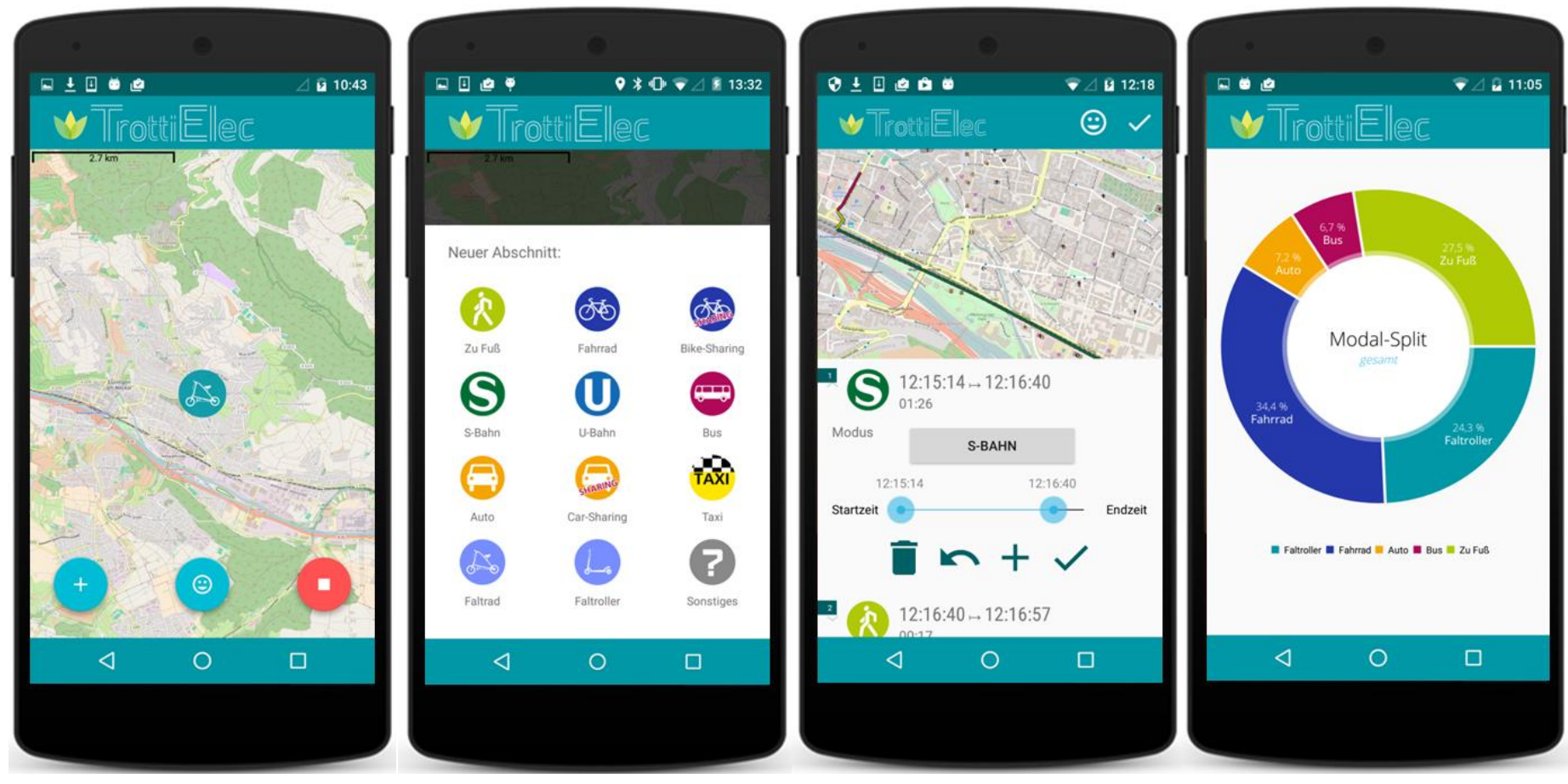

Fig. 2: Smartphone-App to record the proband-specific traffic chains.

The "Mobility Recognizer" is an advanced electronical travel diary, which allows users to collect sensor data automatically during travels. Once enough sensor data has been collected, the Mobility Recognizer can determine the user's transportation mode automatically, by carrying the smartphone in the pocket or rather at the body. At the end of the trip, the proband can validate the trip data and initiates an upload to the university server (or discards the journey).

Following a far-reaching analysis of mobility data across various categories, a machine learning process was developed that assimilates the typical characteristics of collected sensor data for each mode of transport. Automatic transportation mode detection has significant benefits in comparison to conventional travel diaries and questionnaires. It can increase the automation of analyses, which in turn can be less time consuming. Conventional methods also require more effort by the respondents and are less user-friendly. In addition, paper pencil questionnaires are usually filled out after the trip from memory, which can cause inaccurate data collection.

The rapid increase in smartphone usage as well as the increased power efficiency and battery runtimes offers userfriendly and precise opportunity to utilise them to collect travel data. Smartphones nowadays are equipped with a variety of sensors. The most suitable sensors for transportation mode detection are the motion sensors, such as the Accelerometer, which measures the accelerations along three axes or the Gyroscope, which measures the angular speed along three axes of the device. The user's location, the velocity, the direction of movement and the altitude can be obtained by the internal GPS receiver and its sensor framework. The gathered sensor data comprises characteristic patterns for different transportation modes. While power containing measures like the resulting acceleration of the device contain information about activity and thereby can help to distinguish between motorised transport and non-motorised transport, the GPS speed can additionally help to distinguish between modalities, which have similar acceleration patterns.

If geographic coordinates and time schedules of public transport providers are available, the distance from the user to the next bus station and the time where the distance is minimal does indicate if the user probably takes a bus, drives a car 
or takes the city train. Moreover, the heading change rate extracted from the GPS, the angular speed and the amplitudes of fast Fourier transform at certain frequencies are extracted from the sensor values.

To identify these patterns, supervised machine learning techniques are applied. In comparison to unsupervised techniques, supervised machine learning does require learning data, which is sensor data labelled with the respective transportation mode. To classify the collected sensor data among the possible classes like walking, driving a car or driving a TrottiElec, supervised machine learning algorithms, like decision trees and random forest deliver the best results. If the collected data does not contain enough characteristic patterns for each mode or the data is very noisy, decision trees tend to overfit, which results in unprecise prediction, because the model does not describe the underlying model, but reacts to noise. To prevent the classifier from overfitting, bagged trees or random forest should be applied.

This data can be immediately evaluated and shown on the smartphone - users see their own modal split and have the option of rating the level of comfort experienced in each case. In addition, consolidating the data from a wide range of users and combining it with other data sources serves to identify behavioural patterns and anomalies. With the utilisation of the Mobility Recognizer by many respondents, it is now possible to collate, evaluate and visualize multimodal journeys. The Mobility Recognizer can also help to answer entrepreneurial questions, e.g. examining multimodal mobility or determining and evaluating mobility-related factors such as convenience or user satisfaction.

Both of these elements provide essential information to mobility providers to make mobility options and services more user-friendly.

\section{Adaptive Speed Limitation}
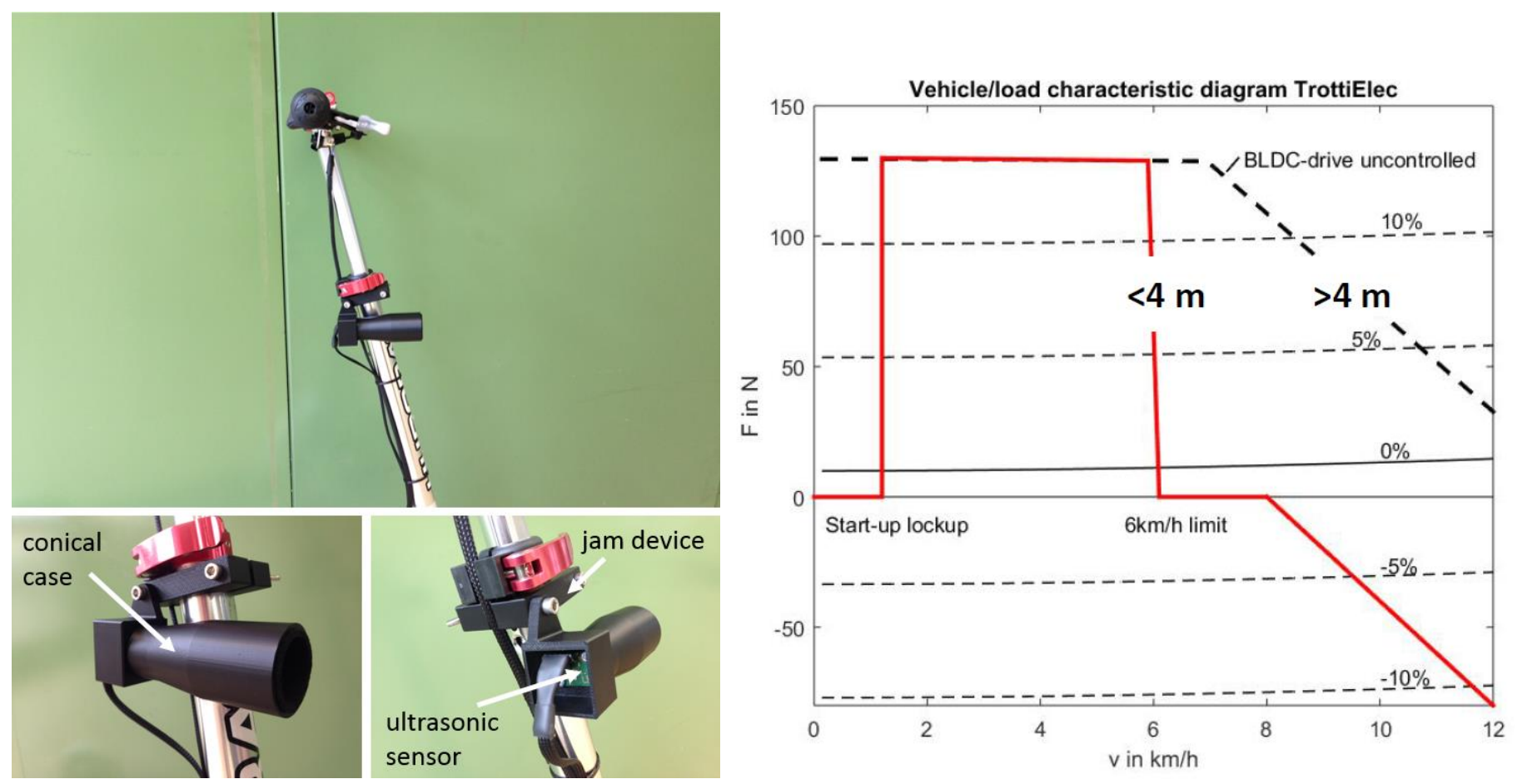

Fig. 3: Adaptive Speed Limitation based on Ultra-sonic Sensors.

The first fleet experiments made clear that the fixed $6 \mathrm{~km} / \mathrm{h}$ speed limit makes the TrottiElecs unattractive compared to the unmotorised scooters due to the missing traveling time advantage on the last mile. In contrast, the unlimited scooter that runs up to $12 \mathrm{~km} / \mathrm{h}$ has been quite more attractive for our probands. To ensure a small relative velocity to the pedestrian traffic participants, we developed an ultrasonic-sensor-based adaptive speed limitation (ASL) that limits the electrical assistance to $6 \mathrm{~km} / \mathrm{h}$ if obstacles are detected within $4 \mathrm{~m}$ range. If no obstacle is detected, the TrottiElec reaches between 10 and $12 \mathrm{~km} / \mathrm{h}$.

Fig. 4 shows the different detection zones of the ultrasonic sensor with and without conical case that has big influence on the laterally width of the detection zone. The grey border-line in Fig. 4 indicates the limit of object detection without 
any adapted case. The laterally width of this detection zone is more than $2 \mathrm{~m}$ and thus not allowing driving in a parallel manner to house walls. The blue and the brown lines show slimmer areas of detection due to the conical case. The controlled zone finally is described by the green area. The maximal lateral controlled distance to an obstacle is $1,20 \mathrm{~m}$. If the scooter is faster than $6 \mathrm{~km} / \mathrm{h}$ and if the ultrasonic distance sensor detects an obstacle in this area, the motor will change to idle run and the scooter reduces speed. When driving on a narrow path the scooter reduces the electrical assistance too.

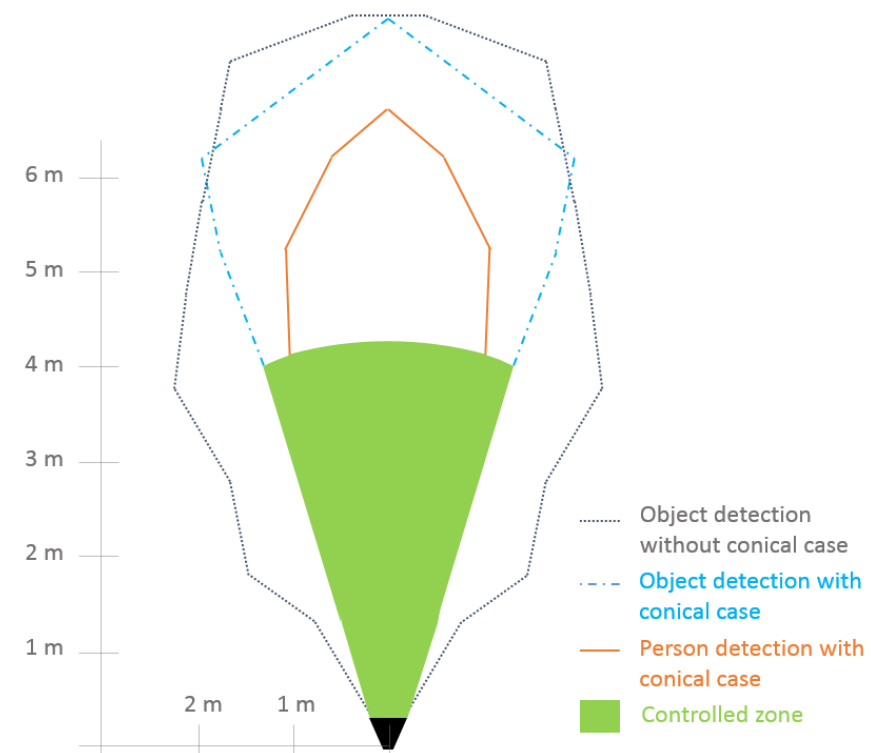

Fig. 4: Detection zones of the ultrasonic sensor with and without conical case.

Fig. 5 shows the velocity comparison between the three proband groups in the first fleet experiment by example of the $1-\mathrm{km}$-distance between university campus and train station. The travelling time afoot is about 15 minutes (without perspiration), the unmotorised scooters require 6 minutes (with significant perspiration on that distance) and the TrottiElecs are slightly faster (5:30 minutes without perspiration). The ASL works well as this can be seen in Fig. 5 at the $350 \mathrm{~m}-$ distance where the trip crosses the crowded pedestrian zone, while the walkway on the other trip segments is more or less free.

This example shows that the TrottiElec with ASL combines both, safety and traffic advantages as well as perspiration free micro-mobility. Thus, slow electrified foldable scooters should be tolerated on walkways as the unmotorised folding scooters are tolerated today. This would be the smallest threshold for a future product. The permission for walkways is part of the ongoing negotiations between the TrottiElec research cooperation and the Ministry of Traffic and Infrastructure Baden-Württemberg.

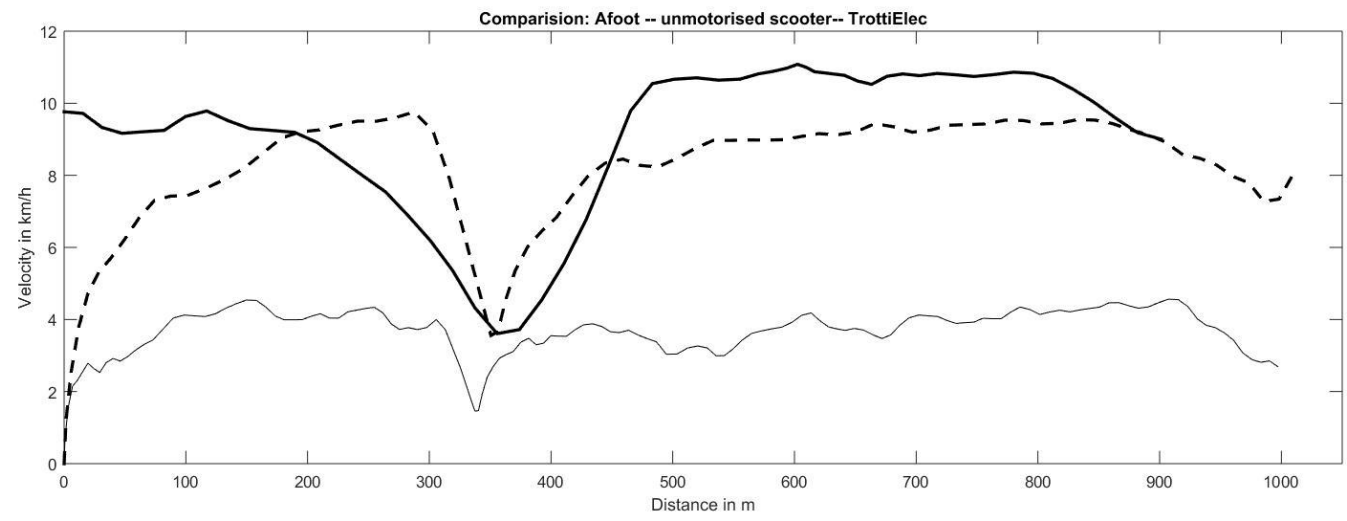

Fig. 5: Exemplary comparision of electrified scooters with ASL and unmotorised scooters on a typical last mile distance (here Esslingen train station to university campus). 


\section{Conclusion}

Slow foldable E-ULV are proven to be a key element for future sustainable public traffic chains. The electrification can be realized by a light and unexpensive add-on kit that can be adapted for several folding scooters. The E-ULV have a significant potential to shift car-kilometers to the public transport if their operating velocity is in the range of unpowered scooters. The main obstacle for a legal applicable electric "last mile scooter" is the missing vehicle category on public walkways. The ongoing fleet experiment will smooth the way to such a future micro-mobility vehicle category or - much better for a future product - increase/ promote the acceptance of electrified folding scooters with adaptive speed limitation on public walkway as "tolerated toys".

\section{Acknowledgements}

This contribution had been worked out in the applied research project "Nachhaltig Mobil - TrottiElec", funded by the Ministry of Traffic and Infrastructure Baden-Württemberg, Stuttgart. The authors thank for the support of TÜV SÜD in Munich/Pforzheim and the support of the industrial Partners Hudora GmbH, Remscheid, and Robert Bosch GmbH, Renningen.

\section{References}

[1] N. N., Petits véhicules motorisés (SUIVI DU CEN/TC 354): AFNOR/S55T prEN16990, http://www2.afnor.org, 2016.

[2] O. Zirn, O. Strobel, A. Norkauer, "TrottiElec - ultraleichter E-Kickroller für geschlossene Wegeketten im urbanen Verkehr," AALE 2014, OTH Regensburg, 8./9.5, 2014.

[3] O. Zirn, M. Rüther, "Foldable Electrified Ultralight Vehicles as Key Component for Sustainable Traffic Chains," 5th International Energy and Sustainability Conference, 2016, IEEE Xplore DOI 10.1109/IESC.2016.7569483.

[4] M. Kagerbauer, T. Hilgert, O. Schroeder, P. Vortisch, "Houshold travel survey of intermodal trips - Approach, challenges and comparison," Transportation Research Procedia, vol. 11, pp. 330-339, 2015. 